\title{
Measure-Theoretic Characterizations of Certain Topological Properties
}

\author{
by \\ D. BUHAGIAR, E. CHETCUTI and A. DVUREČENSKIJ
}

Presented by Czestaw BESSAGA

\begin{abstract}
Summary. It is shown that Čech completeness, ultracompleteness and local compactness can be defined by demanding that certain equivalences hold between certain classes of Baire measures or by demanding that certain classes of Baire measures have non-empty support. This shows that these three topological properties are measurable, similarly to the classical examples of compact spaces, pseudo-compact spaces and realcompact spaces.
\end{abstract}

1. Introduction. This paper is devoted to the measurability of certain topological properties, that is, to measure-theoretic characterizations of some topological properties. Namely, we will show that local compactness, ultracompleteness and Čech completeness are measurable properties. All spaces are assumed to be Tikhonov, that is, $T_{1}$ spaces on which every point $x$ and every closed set $F$ not containing $x$ are functionally separated. Let us denote by $\mathcal{M}(X), \mathcal{M}_{\sigma}(X), \mathcal{M}_{\tau}(X)$, and $\mathcal{M}_{t}(X)$ the sets of all regular measures, $\sigma$-additive measures, $\tau$-additive measures and tight measures on a Tikhonov space $X$ respectively, and by $\mathcal{T}(X), \mathcal{T}_{\sigma}(X), \mathcal{T}_{\tau}(X)$, and $\mathcal{D}(X)$ the sets of all two-valued measures, two-valued $\sigma$-additive measures, two-valued $\tau$-additive measures and Dirac measures on $X$ respectively. Since the publication of the paper of V. S. Varadarajan [17] the above sets of measures have been subjected to much scrutiny. Properties of spaces which can be defined

2000 Mathematics Subject Classification: Primary 28C15, 54D45, 54D99; Secondary $54 \mathrm{E} 15,54 \mathrm{E} 50$.

Key words and phrases: Baire measure, Čech completeness, ultracompleteness, local compactness.

The paper has been partially supported by the Grant VEGA No- $2 / 3163 / 23$ and by Science Technology Assistance Agency under contract No. APVT-51-032002, Bratislava and by the AWRF of the University of Malta. 
by demanding that certain equivalences hold between these sets of measures were studied by J. J. Dijkstra [5], W. Moran [11-13] and others. Classical examples of measurable topological properties are pseudo-compactness, realcompactness and compactness, where the following results are known:

THEOREM 1.1 ([1, 9]). The following conditions are equivalent for a space $X$ :

(i) $X$ is pseudo-compact.

(ii) $\mathcal{M}(X)=\mathcal{M}_{\sigma}(X)$.

(iii) $\mathcal{T}(X)=\mathcal{T}_{\sigma}(X)$.

Theorem $1.2([10]) . X$ is realcompact if, and only if, $\mathcal{T}_{\sigma}(X)=\mathcal{D}(X)$.

THEOREM 1.3 ([1]). The following conditions are equivalent for a space $X$ :

(i) $X$ is compact.

(ii) $\mathcal{M}(X)=\mathcal{M}_{\tau}(X)$.

(iii) $\mathcal{T}(X)=\mathcal{T}_{\tau}(X)$.

(iv) $\mathcal{T}(X)=\mathcal{D}(X)$.

Chapter VIII.4 of J. Nagata [14] serves as a very good short introduction to the subject.

2. Basic definitions and lemmas. Some of the terminology used might be somewhat different from the standard usage and therefore we will give the definitions. All spaces are assumed to be Tikhonov. The abbreviations f.i.p. and c.i.p. stand for finite intersection property and countable intersection property respectively.

Definition 2.1. Let $\mathcal{A}(X)$ be the algebra generated by the collection $Z(X)$ of all zero sets of the space $X$, i.e. the smallest collection $\mathcal{A}(X)$ of subsets of $X$ satisfying

(i) $B_{1} \cap B_{2} \in \mathcal{A}(X)$ whenever $B_{1}, B_{2} \in \mathcal{A}(X)$;

(ii) $X \backslash B \in \mathcal{A}(X)$ whenever $B \in \mathcal{A}(X)$;

(iii) $\mathcal{Z}(X) \subset \mathcal{A}(X)$.

Definition 2.2. By a measure $\mu$ on $\mathcal{A}(X)$ we mean a finitely additive non-negative real-valued function on $\mathcal{A}(X)$.

Definition 2.3. If $\mu$ is a measure on $\mathcal{A}(X)$, then the outer measure $\mu^{*}$ is defined by

$$
\mu^{*}(A)=\inf \{\mu(U): A \subset U \in \mathfrak{C}(X)\} \quad \text { for every } A \subset X,
$$

where $\mathrm{C}(X)$ denotes the collection of all cozero sets of $X$.

Definition 2.4. A measure $\mu$ is called regular if $\mu(B)=\inf \{\mu(U)$ : $B \subset U \in \mathcal{C}(X)\}$ for each $B \in \mathcal{A}(X)$. Equivalently, $\mu$ is regular if $\mu(B)=$ $\sup \{\mu(Z): B \supset Z \in Z(X)\}$ for each $B \in \mathcal{A}(X)$. 
From now on by a measure we mean a regular measure.

Definition 2.5. Let $\mu$ be a measure on $\mathcal{A}(X)$.

(I) $\mu$ is called $\sigma$-additive if

$$
\mu\left(\bigcup_{i=1}^{\infty} B_{i}\right)=\sum_{i=1}^{\infty} \mu\left(B_{i}\right)
$$

whenever $\left\{B_{i}: i=1,2, \ldots\right\}$ is a disjoint countable subcollection of $\mathcal{A}(X)$ with $\bigcup_{i=1}^{\infty} B_{i} \in \mathcal{A}(X)$.

(II) $\mu$ is called $\tau$-additive if for every open cover $\mathcal{U}$ of $X$ by cozero sets and for every $\varepsilon>0$ there is a finite subcollection $\mathcal{V}$ of $\mathcal{U}$ such that $\mu(\bigcup \mathcal{V})>\mu(X)-\varepsilon$

(III) $\mu$ is called tight if for every $\varepsilon>0$ there is a compact subset $C$ of $X$ such that $\mu^{*}(C)>\mu(X)-\varepsilon$.

We denote by $\mathcal{M}(X), \mathcal{M}_{\sigma}(X), \mathcal{M}_{\tau}(X)$ and $\mathcal{M}_{t}(X)$ the sets of all regular measures, $\sigma$-additive measures, $\tau$-additive measures, and tight measures on $X$ respectively.

Proposition 2.1. For any space $X$ we have

$$
\mathcal{M}_{t}(X) \subset \mathcal{M}_{\tau}(X) \subset \mathcal{M}_{\sigma}(X) \subset \mathcal{M}(X) .
$$

A measure $\mu$ on $X$ is called a two-valued measure if $\mu(\mathcal{A}(X))=\{0,1\}$. Let $x$ be a fixed point of $X$. Then a Dirac measure $\delta_{x}$ is defined by

$$
\delta_{x}(B)= \begin{cases}1 & \text { if } x \in B \in \mathcal{A}(X), \\ 0 & \text { if } x \notin B \in \mathcal{A}(X) .\end{cases}
$$

We denote by $\mathcal{T}(X), \mathcal{T}_{\sigma}(X), \mathcal{T}_{\tau}(X), \mathcal{T}_{t}(X)$ and $\mathcal{D}(X)$ the sets of all twovalued measures, two-valued $\sigma$-additive measures, two-valued $\tau$-additive measures, two-valued tight measures and Dirac measures on $X$ respectively.

Proposition 2.2. For any space $X$ we have

$$
\mathcal{T}_{t}(X)=\mathcal{T}_{\tau}(X)=\mathcal{D}(X) \subset \mathcal{T}_{\sigma}(X) \subset \mathcal{T}(X) .
$$

Definition 2.6. Let $\mu$ be a measure on $X$. Then by the support of $\mu$ we mean the set

$$
S(\mu)=\bigcap\{Z \in \mathcal{Z}(X): \mu(Z)=\mu(X)\}=X \backslash \bigcup\{U \in \mathcal{C}(X): \mu(U)=0\} .
$$

We need the following lemmas (see for example [5]). By a zero filter of a space $X$ we mean a filter in $Z(X)$.

Lemma 2.3. Let $\mathcal{F}$ be a maximal zero filter of a space $X$. Then the map $\mu: \mathcal{A}(X) \rightarrow\{0,1\}$ defined by $\mu(B)=1$ if, and only if, there exists $Z \in \mathcal{F}$ with $Z \subset B$ is an element of $\mathcal{T}(X)$. Moreover, if $\mathcal{F}$ has c.i.p., then $\mu$ is an element of $\mathcal{T}_{\sigma}(X)$. 
Lemma 2.4. Let $\mu: \mathcal{A}(X) \rightarrow\{0,1\}$ be an element of $\mathcal{T}(X)$. Then the collection $\mathcal{F}=\{Z \in \mathcal{Z}(X): \mu(Z)=1\}$ defines a maximal zero filter of the space $X$. Moreover, if $\mu$ is an element of $\mathcal{T}_{\sigma}(X)$, then $\mathcal{F}$ has c.i.p.

\section{Measurability of Čech completeness, ultracompleteness and} local compactness. In this section we give measure-theoretic criteria for Čech complete spaces, ultracomplete spaces and locally compact spaces.

Definition 3.1. Let $\mathcal{U}$ be a cozero cover of a space $X$ and $\mu$ a measure on $X$. Then $\mu$ is said to be $\mathcal{U}$-positive if there exists a $U \in \mathcal{U}$ such that $\mu(U)>0$.

If $\mathfrak{U}$ is a collection of cozero covers, then $\mu$ is said to be $\mathfrak{U}$-positive if $\mu$ is $\mathcal{U}$-positive for every $\mathcal{U} \in \mathfrak{U}$.

REMARK 3.1. One can easily see that any $\tau$-additive measure (and therefore any tight measure) is $\mathcal{U}$-positive for any cozero cover $\mathcal{U}$ of $X$. Consequently, any $\mu \in \mathcal{M}_{\tau}(X)$ (or $\in \mathcal{M}_{t}(X)$ ) and any Dirac measure is $\mathfrak{U}$-positive for any $\mathfrak{U}$.

Definition 3.2. For a collection $\mathfrak{U}$ of cozero covers of $X$ we denote by $\mathcal{M}(X, \mathfrak{U})$ (resp. $\mathcal{M}_{\sigma}(X, \mathfrak{U})$ ) the set of $\mathfrak{U}$-positive measures in $\mathcal{M}(X)$ (resp. $\left.\mathcal{M}_{\sigma}(X)\right)$. Similarly, $\mathfrak{T}(X, \mathfrak{U})$ (resp. $\mathcal{T}_{\sigma}(X, \mathfrak{U})$ ) is the set of $\mathfrak{U}$-positive measures in $\mathcal{T}(X)\left(\operatorname{resp} . \mathcal{T}_{\sigma}(X)\right)$.

Recall that a space $X$ is said to be $\check{C}$ ech complete if it is a $G_{\delta}$-set in one (equivalently, in all) of its Hausdorff compactifications [4]. By a result of E. Čech [4], a metrizable space is completely metrizable (i.e., metrizable by a complete metric, a notion defined by M. Fréchet in 1906 [7]) if and only if it is Čech complete. The following internal characterization of Čech complete spaces was established independently by Z. Frolík in 1960 [8] and by A. V. Arkhangel'skiĩ in 1961 [2].

Proposition $3.1([8,2])$. A Tikhonov space $X$ is Čech complete if, and only if, there is a sequence $\mathfrak{U}=\left\{\mathcal{U}_{n}: n \in \mathbb{N}\right\}$ of open covers of $X$ such that every $\mathfrak{U}$-Cauchy filter base $\mathcal{F}$ on $X$ clusters in $X$, where $\mathcal{F}$ is said to be $\mathfrak{U}$-Cauchy if for every $\mathcal{U} \in \mathfrak{U}$ there exists some $U \in \mathcal{U}$ such that $F \subset U$ for some $F \in \mathcal{F}$.

We will need the following slight modification of Proposition 3.1, the proof of which slightly differs from that of Proposition 3.1 given in [6, Theorem 3.9.2].

Corollary 3.2. A space $X$ is Čech complete if, and only if, there exists a sequence $\mathfrak{U}$ of cozero covers of $X$ such that every zero $\mathfrak{U}$-Cauchy filter has a non-empty intersection. 
Proof. We only need to proof sufficiency. Assume that the space $X \subset \beta X$ has a sequence $\mathfrak{U}=\left\{\mathcal{U}_{n}: n \in \mathbb{N}\right\}$ of cozero covers with the said property. Let $\mathcal{U}_{n}=\left\{U_{\alpha}^{n}: \alpha \in \Lambda_{n}\right\}$ for every $n \in \mathbb{N}$ and let $V_{\alpha}^{n}$ be an open subset of $\beta X$ with $U_{\alpha}^{n}=V_{\alpha}^{n} \cap X$ for every $\alpha \in \Lambda_{n}$ and $n \in \mathbb{N}$. Evidently,

$$
X \subset \bigcap_{n \in \mathbb{N}} \bigcup_{\alpha \in \Lambda_{n}} V_{\alpha}^{n}
$$

and to prove that $X$ is Čech complete we need to show that the reverse inclusion also holds.

Take any $x \in \bigcap_{n \in \mathbb{N}} \bigcup_{\alpha \in \Lambda_{n}} V_{\alpha}^{n}$ and let $\mathcal{N}(x)$ be the collection of open neighborhoods of $x$ in $\beta X$. Consider the collection $\mathcal{F}=\{X \cap \bar{V}: V \in \mathcal{N}(x)\}$, where $\bar{V}$ is the closure of $V$ in $\beta X$. Note that $\mathcal{F}$ is a closed filter base in $X$. Now if $x \in V \in \mathcal{N}(x)$, then by regularity of $\beta X$, there exists $V^{\prime} \in \mathcal{N}(x)$ such that $x \in V^{\prime} \subset \overline{V^{\prime}} \subset V$. Next, by normality of $\beta X$, there exists a zero set $W$ in $\beta X$ such that $\overline{V^{\prime}} \subset W \subset V$ and $W \cap X$ is a zero set in $X$. Let $\mathcal{F}^{\prime}$ be the zero filter in $X$ generated by $\mathcal{F}$. We have just seen that $\bigcap \mathcal{F}=\bigcap \mathcal{F}^{\prime}$. Since for any $n \in \mathbb{N}$ there exists $\alpha \in \Lambda_{n}$ with $x \in V_{\alpha}^{n}$, it follows from regularity of $\beta X$ that $\mathcal{F}$, and hence $\mathcal{F}^{\prime}$, is $\mathfrak{U}$-Cauchy. By our assumption, $X \cap \cap \mathcal{F}^{\prime} \neq \emptyset$ and since $\bigcap\{\bar{V}: V \in \mathcal{N}(x)\}=\{x\}$, we conclude that $x \in X$.

THEOREM 3.3. The following conditions are equivalent for a space $X$ :

(i) $X$ is Čech complete.

(ii) There exists a sequence $\mathfrak{U}$ of cozero covers of $X$ such that every $\mathfrak{U}$-positive two-valued measure on $X$ has a non-empty support.

(iii) There exists a sequence $\mathfrak{U}$ of cozero covers of $X$ such that $\mathfrak{T}(X, \mathfrak{U})=$ $\mathcal{T}_{\tau}(X, \mathfrak{U})$.

Proof. (i) $\Rightarrow$ (ii). Let $X$ be Čech complete and let $\mathfrak{U}=\left\{\mathcal{U}_{i}: i \in \mathbb{N}\right\}$ be a sequence of cozero covers of $X$ such that every zero $\mathfrak{U}$-Cauchy filter has a non-empty intersection. Let $\mu \in \mathcal{T}(X, \mathfrak{U})$ and $\mathcal{F}=\{Z \in \mathcal{Z}(X): \mu(Z)=1\}$. By Lemma $2.4, \mathcal{F}$ is a maximal zero filter of the space $X$ and it is not difficult to see that it is $\mathfrak{U}$-Cauchy and so has non-empty intersection. If $x \in \cap \mathcal{F}$ then for every $U \in \mathcal{C}(X)$ with $x \in U$, we have $\mu(U)=1$ so that $\mu$ has a non-empty support.

(ii) $\Rightarrow$ (iii). Let there exist a sequence $\mathfrak{U}$ of cozero covers of $X$ such that the trivial measure 0 is the only $\mathfrak{U}$-positive two-valued measure on $X$ with an empty support. Assume that there is a measure $\mu \in \mathcal{T}(X, \mathfrak{U})$ which is not in $\mathcal{T}_{\tau}(X, \mathfrak{U})$. Then there exists a cozero cover $\mathcal{V}$ of $X$ such that $\mu\left(\bigcup_{i=1}^{n} V_{i}\right)=0$ for every finite subcollection $V_{1}, \ldots, V_{n}$ of $\mathcal{V}$. In particular, $\mu(V)=0$ for every $V \in \mathcal{V}$ and therefore $\mu$ has an empty support so that $\mu=0$.

(iii) $\Rightarrow\left(\right.$ i). Let $\mathfrak{U}=\left\{\mathcal{U}_{i}: i \in \mathbb{N}\right\}$ be a sequence of cozero covers of $X$ such that $\mathfrak{T}(X, \mathfrak{U})=\mathcal{T}_{\tau}(X, \mathfrak{U})$. We show that $\mathfrak{U}$ is a Cauchy complete sequence of cozero covers. Assume that it is not; then there exists a zero $\mathfrak{U}$-Cauchy 
filter with an empty intersection. Let $\mathcal{F}^{\prime}$ be a maximal zero filter on $\mathcal{F}$ and construct $\mu \in \mathcal{T}(X)$ by Lemma 2.3, i.e. $\mu(Y)=1$ if, and only if, there exists $F \in \mathcal{F}^{\prime}$ with $F \subset Y$. Then $\mu$ is $\mathfrak{U}$-positive since $\mathcal{F}$ (and so $\mathcal{F}^{\prime}$ ) is $\mathfrak{U}$-Cauchy. By assumption, $\bigcap \mathcal{F}^{\prime}=\emptyset$ and therefore, $\mathcal{V}=\left\{X \backslash F: F \in \mathcal{F}^{\prime}\right\}$ is a cozero cover of $X$. Since $\mu(V)=0$ for every $V \in \mathcal{V}$, we have $\mu\left(\bigcup \mathcal{V}^{\prime}\right)=0$ for every finite subcollection $\mathcal{V}^{\prime}$ of $\mathcal{V}$. Consequently, $\mu$ is not $\tau$-additive, a contradiction.

Similarly one can prove the following result for uniform spaces. By a uniformity $\mathfrak{U}$ we mean a uniformity given in terms of coverings.

COROLlaRY 3.4. The following conditions are equivalent for a uniform space $(X, \mathfrak{U})$ :

(i) $X$ is complete.

(ii) $\mathcal{T}(X, \mathfrak{U})=\mathcal{T}_{\tau}(X, \mathfrak{U})$.

(iii) Every $\mathfrak{U}$-positive two-valued measure on $X$ has a non-empty support.

A similar result can be stated for metric spaces. Indeed, if $(X, \varrho)$ is a metric space, then a measure $\mu \in \mathcal{N}(X)$ is said to live on arbitrarily small sets if for every $\varepsilon>0$ there exists a set $Y \subset X$ such that $\operatorname{diam}(Y)<\varepsilon$ and $\mu(Y)>0$. We then have the following result.

COROLlaRY 3.5. The following conditions are equivalent for a metric space $(X, \varrho)$ :

(i) $X$ is complete.

(ii) Every two-valued measure on $X$ that lives on arbitrarily small sets is $\tau$-additive.

(iii) Every two-valued measure on $X$ that lives on arbitrarily small sets has a non-empty support.

A space $X$ is said to be ultracomplete if it has countable character in some (equivalently, in every) compactification $c X$ of the space $X$, i.e. $\chi(X, c X)$ $\leq \omega_{0}$. It is clear from the definition that we have the following implications:

$$
\text { locally compact } \Rightarrow \text { ultracomplete } \Rightarrow \text { Čech complete. }
$$

Examples show that none of the above implications are reversible, even in the realm of metrizable spaces (see $[3,15,16]$ ).

The following theorem was proved in [3]. For a collection $\mathcal{P}$ of subsets of a set $X$, we denote by $\mathcal{P}^{F}$ the collection of all unions of finite subcollections from $\mathcal{P}$.

TheORem $3.6([3])$. For every Tikhonov space $X$ the following conditions are equivalent:

(i) $X$ is ultracomplete. 
(ii) There exists a sequence $\left\{\mathcal{U}_{n}: n \in \mathbb{N}\right\}$ of open covers of $X$ such that, if $\mathcal{F}$ is a filter base on $X$ which meshes with some sequence $\left\{U_{n}: U_{n} \in \mathcal{U}_{n}\right\}$, then $\mathcal{F}$ clusters in $X$.

(iii) There exists a sequence $\left\{\mathcal{U}_{n}: n \in \mathbb{N}\right\}$ of open covers of $X$ such that, for every open cover $\mathcal{V}$ of $X$ there exists an $n \in \mathbb{N}$ satisfying $\mathcal{U}_{n}<\mathcal{V}^{F}$, that is, $\mathcal{U}_{n}$ refines $\mathcal{V}^{F}$.

Ultracomplete spaces as defined above were termed strongly complete spaces by V. I. Ponomarev and V. V. Tkachuk in [15] and item (ii) in the above theorem was given as a definition for cofinally Cech complete spaces by S. Romaguera in [16].

Before we give a characterization of ultracomplete spaces in terms of measures we need the following result on compact spaces. The result is similar to a characterization result for measure-compact spaces given by W. Moran [11].

THEOREM 3.7. The following conditions are equivalent for a space $X$ :

(i) $X$ is compact.

(ii) The trivial measure 0 is the only measure on $X$ with an empty support.

(iii) The trivial measure 0 is the only two-valued measure on $X$ with an empty support.

Proof. (i) $\Rightarrow$ (ii). Assume that $X$ is compact and $\mu \in \mathcal{M}(X)\left(=\mathcal{M}_{\tau}(X)\right)$ has an empty support. Then there exists a cozero cover $\mathcal{U}=\left\{V_{\alpha}: \alpha \in \Lambda\right\}$ such that $\mu\left(V_{\alpha}\right)=0$ for every $\alpha \in \Lambda$. For every positive real number $\varepsilon$, there exists a finite subcover $V_{\alpha_{1}}, \ldots, V_{\alpha_{n}}$ satisfying $\mu\left(\bigcup_{i=1}^{n} V_{\alpha_{i}}\right)>\mu(X)-\varepsilon$. But $\mu\left(\bigcup_{i=1}^{n} V_{\alpha_{i}}\right) \leq \sum_{i=1}^{n} \mu\left(V_{\alpha_{i}}\right)=0$, so that $\mu(X)=0$ and $\mu$ is the trivial measure.

(ii) $\Rightarrow$ (iii) is obvious.

(iii) $\Rightarrow$ (i). Assume that $X$ is not compact and $\mathcal{F}$ is a maximal zero filter which does not converge. Define $\mu \in \mathcal{T}(X)$ by use of Lemma 2.3 and $\mathcal{F}$. For every $x \in X$ there exists a cozero neighborhood $V$ of $x$ such that $V \cap F_{V}=\emptyset$ for some $F_{V} \in \mathcal{F}$, and therefore, $\mu(V)=0$. Consequently, the support of $\mu$ is empty and $\mu$ is not the trivial measure.

We now give a measure-theoretic characterization for ultracomplete spaces.

THEOREM 3.8. The following conditions are equivalent for a space $X$ :

(i) $X$ is ultracomplete.

(ii) There exists a sequence $\mathfrak{U}$ of cozero covers of $X$ such that every $\mathfrak{U}$ positive measure on $X$ has a non-empty support.

Proof. (i) $\Rightarrow$ (ii). Suppose $X$ is ultracomplete and let $\mathfrak{U}=\left\{\mathcal{U}_{i}: i \in \mathbb{N}\right\}$ be a sequence of cozero covers of $X$ satisfying property (iii) of Theorem 3.6. 
Let $\mu$ be a $\mathfrak{U}$-positive measure on $X$ with an empty support. Then there exists a cozero cover $\mathcal{V}$ of $X$ such that $\mu(V)=0$ for every $V \in \mathcal{V}$. By our assumption on $\mathfrak{U}$, there exists an $i \in \mathbb{N}$ such that $\mathcal{U}_{i}<\mathcal{V}^{F}$ and since $\mu$ is $\mathfrak{U}$-positive, there exists $U \in \mathcal{U}_{i}$ such that $\mu(U)>0$. One can find $\widehat{V} \in \mathcal{V}^{F}$ such that $U \subset \widehat{V}=\sum_{k=1}^{n} V_{k}$, where $V_{k} \in \mathcal{V}$ for all $k=1, \ldots, n$. Thus $\mu(U) \leq \sum_{k=1}^{n} \mu\left(V_{k}\right)=0$, a contradiction.

(ii) $\Rightarrow$ (i). Let there exist a sequence $\mathfrak{U}=\left\{\mathcal{U}_{i}: i \in \mathbb{N}\right\}$ of cozero covers of $X$ such that every $\mathfrak{U}$-positive measure on $X$ has a non-empty support and assume that $X$ is not ultracomplete. For every $i \in \mathbb{N}$ and every $x \in X$ there exist a cozero set $V_{x}^{i}$, a zero set $Z_{x}^{i}$ and $U_{x}^{i} \in \mathcal{U}_{i}$ such that $x \in V_{x}^{i} \subset \overline{V_{x}^{i}} \subset$ $Z_{x}^{i} \subset U_{x}^{i}$. Let $\mathcal{V}_{i}=\left\{V_{x}^{i}: x \in X\right\}$ for every $i \in \mathbb{N}$, so that $\mathcal{V}_{i}<\mathcal{U}_{i}$, and let $\mathfrak{V}=\left\{\mathcal{V}_{i}: i \in \mathbb{N}\right\}$. Since $X$ is not ultracomplete there exists a cozero cover $\mathcal{W}$ of $X$ such that $\mathcal{V}_{i} \nless \mathcal{W}^{F}$ for every $i \in \mathbb{N}$, that is, there exists $V^{i} \in \mathcal{V}_{i}$ such that $V^{i} \cap(X \backslash O) \neq \emptyset$ for every $O \in \mathcal{W}^{F}$.

Since $X$ is not compact, by Theorem 3.7 there exists a measure $\mu \in \mathcal{T}(X)$ with an empty support, that is, there exists a cozero cover $\mathcal{V}$ of $X$ such that $\mu(V)=0$ for every $V \in \mathcal{V}$. Let $\mathcal{O}=\mathcal{V} \wedge \mathcal{W}$. Then $\mathcal{V}_{i} \nless \mathcal{O}^{F}$ for every $i \in \mathbb{N}$ and $\mu(O)=0$ for every $O \in \mathcal{O}$. Take any $i \in \mathbb{N}$. Since $\mathcal{V}_{i} \nless \mathcal{O}^{F}$, there exists $V^{i}=V_{x}^{i} \in \mathcal{V}_{i}$ such that $V_{x}^{i} \cap(X \backslash O) \neq \emptyset$ for every $O \in \mathcal{O}^{F}$ and therefore, $Z_{x}^{i} \cap(X \backslash O) \neq \emptyset$ for every $O \in \mathcal{O}^{F}$. Consider $\mathcal{F}_{i}=\left\{Z_{x}^{i} \cap(X \backslash O): O \in \mathcal{O}^{F}\right\}$. Then $\mathcal{F}_{i}$ has the f.i.p., consists of zero sets in $X$ and $\bigcap \mathcal{F}_{i}=\emptyset$. Let $\mathcal{F}_{i}^{\prime}$ be a maximal zero filter on $\mathcal{F}_{i}$ in $X$ and construct $\mu_{i} \in \mathcal{T}(X)$ by Lemma 2.3, i.e. $\mu_{i}(Y)=1$ if, and only if, there exists $F \in \mathcal{F}_{i}^{\prime}$ with $F \subset Y$. Finally, we denote $\mu$ by $\mu_{0}$ and we let $m=\sum_{i=0}^{\infty} 2^{-i} \mu_{i}$. Then $m(A) \leq 2$ for every $A \in \mathcal{A}(X)$.

We next show that $m$ has an empty support. Take any $O \in \mathcal{O}$. Since $X \backslash O$ is a zero set and is in $\mathcal{F}_{i}^{\prime}$ for every $i \in \mathbb{N}$, we have $\mu_{i}(X \backslash O)=1$ and therefore $\mu_{i}(O)=0$. Also, as noted above, $\mu_{0}(O)=0$. Consequently, $m(O)=0$ for every $O \in \mathcal{O}$, which shows that $m$ has an empty support. Since $\mu_{i}\left(Z_{x}^{i}\right)=1$, there exists $U_{x}^{i} \in \mathcal{U}_{i}$ such that $\mu_{i}\left(U_{x}^{i}\right)=1$ so that $m\left(U_{x}^{i}\right)>0$ and $m$ is $\mathfrak{U}$-positive.

We are only left to show that $m \in \mathcal{M}(X)$. Since each $\mu_{i}, i=0,1,2, \ldots$, is additive, it is evident that $m$ is additive. We now show that $m$ is regular. Let $A \in \mathcal{A}(X)$ and let $\varepsilon>0$. Consider $m(A)=\sum_{i=0}^{\infty} 2^{-i} \mu_{i}(A)$. There exists $N \in \mathbb{N}$ such that $\sum_{i=0}^{N} 2^{-i} \mu_{i}(A)>m(A)-\varepsilon / 2$. For every $i=0, \ldots, N$, there exists $Z_{i} \in Z(X)$ such that $Z_{i} \subset A$ and $\mu_{i}\left(Z_{i}\right)>\mu_{i}(A)-\varepsilon / 2$. Let $Z=\bigcup_{i=0}^{N} Z_{i} \in Z(X)$. Then $Z \subset A$ and $m(Z) \geq \sum_{i=0}^{N} \frac{1}{2^{i}} \mu_{i}(Z)>\sum_{i=0}^{N} \frac{1}{2^{i}}\left(\mu_{i}(A)-\frac{\varepsilon}{2}\right)>\sum_{i=0}^{N} \frac{1}{2^{i}} \mu_{i}(A)-\frac{\varepsilon}{2}>m(A)-\varepsilon$. Consequently, $m(A)=\sup \{\mu(Z): Z \subset A, Z \in Z(X)\}$ and $m$ is regular.

Similarly one can prove the following result for uniform spaces. 
COROLlary 3.9. The following conditions are equivalent for a uniform space $(X, \mathfrak{U})$ :

(i) $X$ is ultracomplete (cofinally Čech complete).

(ii) Every $\mathfrak{U}$-positive measure on $X$ has a non-empty support.

Finally, we give a measure-theoretic characterization for locally compact spaces.

THEOREM 3.10. The following conditions are equivalent for a space $X$ :

(i) $X$ is locally compact.

(ii) There exists a cozero cover $\mathcal{U}$ of $X$ such that every $\mathcal{U}$-positive measure on $X$ has a non-empty support.

(iii) There exists a cozero cover $\mathcal{U}$ of $X$ such that every $\mathcal{U}$-positive twovalued measure on $X$ has a non-empty support.

(iv) There exists a cozero cover $\mathcal{U}$ of $X$ such that $\mathfrak{T}(X, \mathcal{U})=\mathcal{T}_{\tau}(X, \mathcal{U})$.

Proof. (i) $\Rightarrow$ (ii). Let $X$ be locally compact. For every $x \in X$ there exists a cozero neighborhood $U_{x}$ of $x$ such that $\bar{U}_{x}$ is compact. Let $\mathcal{U}=\left\{U_{x}: x \in X\right\}$ and let $\mu$ be a $\mathcal{U}$-positive measure with an empty support. There exists a cozero cover $\mathcal{V}$ of $X$ such that $\mu(V)=0$ for every $V \in \mathcal{V}$. Since each $\bar{U}_{x}$ is compact, we see that $\mathcal{U}<\mathcal{V}^{F}$ and therefore, $\mu\left(U_{x}\right)=0$ for every $x \in X$, contradicting the fact that $\mu$ is $\mathcal{U}$-positive.

(ii) $\Rightarrow$ (iii) is obvious.

(iii) $\Rightarrow$ (iv). Let there exist a cozero cover $\mathcal{U}$ of $X$ such that the trivial measure 0 is the only $\mathcal{U}$-positive two-valued measure on $X$ with an empty support. Assume that there is a measure $\mu \in \mathfrak{T}(X, \mathcal{U})$ which is not in $\mathcal{T}_{\tau}(X, \mathcal{U})$. Then there exists a cozero cover $\mathcal{V}$ of $X$ such that $\mu\left(\bigcup_{i=1}^{n} V_{i}\right)=0$ for every finite subcollection $V_{1}, \ldots, V_{n}$ of $\mathcal{V}$. In particular, $\mu(V)=0$ for every $V \in \mathcal{V}$ and therefore $\mu$ has an empty support so that $\mu=0$.

(iv) $\Rightarrow$ (i). Let $\mathcal{U}$ be a cozero cover of $X$ such that $\mathcal{T}(X, \mathfrak{U})=\mathcal{T}_{\tau}(X, \mathfrak{U})$. For every $x \in X$ there exists a $U \in \mathcal{U}$ such that $x \in U$ and also a cozero set $V_{x}$ and a zero set $Z_{x}$ such that $x \in V_{x} \subset \bar{V}_{x} \subset Z_{x} \subset U$. Let $\mathcal{V}=\left\{V_{x}: x \in X\right\}$. We prove that $\bar{V}_{x}$ is compact for all $x \in X$ by showing that $Z_{x}$ is compact for all $x \in X$. Indeed, suppose there is an $x \in X$ such that $Z_{x}$ is not compact. Then there is a maximal zero filter $\mathcal{F}$ in $Z_{x}$ with an empty intersection. Let $\mathcal{G}$ be the subfamily of $\mathcal{F}$ defined by $\mathcal{G}=\{Z \in \mathcal{F}: Z$ is a zero set in $X\}$. Then $\mathcal{G}$ has the f.i.p. and is non-empty. Let $\mathcal{F}^{\prime}$ be a maximal zero filter on $\mathcal{G}$ in $X$ and construct $\mu \in \mathcal{T}(X)$ by Lemma 2.3, i.e. $\mu(Y)=1$ if, and only if, there exists $F \in \mathcal{F}^{\prime}$ with $F \subset Y$. Note that $Z_{x} \in \mathcal{F}^{\prime}$ so that $\mu$ is $\mathcal{U}$-positive since $\mu(U)=1$, where $Z_{x} \subset U$ and $U \in \mathcal{U}$. We next show that $\bigcap \mathcal{F}^{\prime}=\emptyset$. Indeed, if $\mathcal{F}^{\prime} \neq \emptyset$ and $y \in \bigcap \mathcal{F}^{\prime}$ then $y \in Z_{x}$. But $\bigcap \mathcal{F}=\emptyset$ so that there exists some $F \in \mathcal{F}$ such that $y \notin F$. There exists a cozero set $W_{y}$ in $X$ such that $y \in W_{y}$ and $W_{y} \cap F=\emptyset$. The set $Z=X \backslash W_{y}$ is a 
zero set in $X$ and $F \subset Z$, so that $Z \in \mathcal{F}^{\prime}$ (since $Z \cap Z_{x} \in \mathcal{G}$ ). Consequently, $\mathcal{V}=\left\{X \backslash F: F \in \mathcal{F}^{\prime}\right\}$ is a cozero cover of $X$. Since $\mu(V)=0$ for every $V \in \mathcal{V}$, we see that $\mu\left(\cup \mathcal{V}^{\prime}\right)=0$ for every finite subcollection $\mathcal{V}^{\prime}$ of $\mathcal{V}$. Therefore $\mu$ is not $\tau$-additive, a contradiction.

In relation to Theorems 3.8 and 3.10 one can pose the following problem:

Problem 3.11. Evidently, if there exists a sequence $\mathfrak{U}$ of cozero covers (resp. one cozero cover $\mathcal{U}$ ) of $X$ such that $\mathcal{M}(X, \mathfrak{U})=\mathcal{M}_{\tau}(X, \mathfrak{U})$ (resp. $\mathcal{M}(X, \mathcal{U})=\mathcal{M}_{\tau}(X, \mathcal{U})$ ) then the same sequence $\mathfrak{U}$ (resp. cozero cover $\left.\mathcal{U}\right)$ is such that every $\mathfrak{U}$-positive (resp. $\mathcal{U}$-positive) measure on $X$ has a non-empty support and so $X$ is ultracomplete (resp. locally compact). Does the converse hold?

The answer to the above question is in the negative, in fact we have the following result.

THEOREM 3.12. Let $\mathfrak{U}$ be any collection of cozero covers of a space $X$. Then $\mathcal{M}(X, \mathfrak{U})=\mathcal{M}_{\tau}(X, \mathfrak{U})$ if, and only if, $X$ is compact.

Proof. We only need to prove the necessity. Suppose $X$ is not compact. There exists a cozero cover $\mathcal{V}$ of $X$ with no finite subcover. Let

$$
\mathcal{F}=\left\{X \backslash \bigcup \mathcal{V}^{\prime}: \mathcal{V}^{\prime} \text { is a finite subcollection of } \mathcal{V}\right\}
$$

Then $\bigcap \mathcal{F}=\emptyset$. Let $\mathcal{F}^{\prime}$ be a maximal zero filter on $\mathcal{F}$ in $X$ and construct $\mu \in \mathcal{T}(X)$ by Lemma 2.3, i.e. $\mu(Y)=1$ if, and only if, there exists $F \in \mathcal{F}^{\prime}$ with $F \subset Y$. Note that $\mu(V)=0$ for every $V \in \mathcal{V}$ and $\mu(X)=1$. Take any Dirac measure $\delta_{x}$, where $x \in X$. Then $\delta_{x} \in \mathcal{M}(X, \mathfrak{U})=\mathcal{M}_{\tau}(X, \mathfrak{U})$. Consider the measure $m=\delta_{x}+\mu \in \mathcal{M}(X, \mathfrak{U})$. On the one hand, $m \in$ $\mathcal{M}_{\tau}(X, \mathfrak{U})$. On the other hand, for every finite subcollection $\mathcal{V}^{\prime}$ of $\mathcal{V}$ we have $\delta_{x}\left(\bigcup \mathcal{V}^{\prime}\right) \leq \delta_{x}(X)=1$ and $\mu\left(\bigcup \mathcal{V}^{\prime}\right)=0$ while $m\left(\bigcup \mathcal{V}^{\prime}\right) \leq 1$. Therefore, $\sup \left\{m\left(\bigcup_{\mathcal{V}^{\prime}}\right): \mathcal{V}^{\prime}\right.$ is a finite subcollection of $\left.\mathcal{V}\right\} \leq 1<m(X)=2$, so that $m \notin \mathcal{M}_{\tau}(X, \mathfrak{U})$.

\section{References}

[1] A. D. Alexandroff, Additive set-functions in abstract spaces, Mat. Sb. 8 (50) (1940), 307-348, 9 (51) (1941), 563-628, 13 (55) (1943), 169-238.

[2] A. V. Arkhangel'skiř, On topological spaces which are complete in the sense of Čech, Vestnik Moskov. Univ. Ser. I Mat. Mekh. 1961, no. 2, 37-40 (in Russian).

[3] D. Buhagiar and I. Yoshioka, Ultracomplete topological spaces, Acta Math. Hungar. 92 (2001), 19-26.

[4] E. Cech, On bicompact spaces, Ann. of Math. 38 (1937), 823-844.

[5] J. J. Dijkstra, Measures in topology, Master Thesis, Univ. of Amsterdam, 1977.

[6] R. Engelking, General Topology, rev. ed., Heldermann, Berlin, 1989.

[7] M. Fréchet, Sur quelques points du calcul fonctionnel, Rend. Circ. Mat. Palermo 22 (1906), 1-74. 
[8] Z. Frolík, Generalization of the $G_{\delta}$-property of complete metric spaces, Czechoslovak Math. J. 10 (1960), 359-379.

[9] I. Glicksberg, The representation of functionals by integrals, Duke Math. J. 19 (1952), 253-261.

[10] E. Hewitt, Linear functionals on spaces of continuous functions, Fund. Math. 37 (1950), 161-189.

[11] W. Moran, The additivity of measures on completely regular spaces, J. London Math. Soc. 43 (1968), 633-639.

[12] -, Measures and mappings on topological spaces, Proc. London Math. Soc. 19 (1969), 493-508.

[13] —, Measures on metacompact spaces, ibid. 20 (1970), 507-524.

[14] J. Nagata, Modern General Topology, 2nd rev. ed., Elsevier, Amsterdam, 1985.

[15] V. I. Ponomarev and V. V. Tkachuk, The countable character of $X$ in $\beta X$ versus the countable character of the diagonal in $X \times X$, Vestnik Moskov. Univ. Ser. I Mat. Mekh. 1987, no. 5, 16-19 (in Russian).

[16] S. Romaguera, On cofinally complete metric spaces, Quest. Answers Gen. Topology 16 (1998), 165-169.

[17] V. S. Varadarajan, Measures on topological spaces, Amer. Math. Soc. Transl. Ser. II 48 (1965), 161-228.

David Buhagiar

Department of Mathematics

Faculty of Science

University of Malta

Msida MSD.06, Malta

E-mail: david.buhagiar@um.edu.mt
Emmanuel Chetcuti and Anatolij Dvurečenskij Mathematical Institute Slovak Academy of Sciences Štefánikova 49

SK-814 73 Bratislava, Slovakia E-mail: chetcuti@mat.savba.sk dvurecen@mat.savba.sk

Received April 15, 2005;

received in final form April 21, 2005 